\title{
Ovarian survival after pelvic radiation: transposition until the age of 35 years
}

\author{
Ellen J. Hoekman ${ }^{1}(1) \cdot$ Dan Knoester $^{1} \cdot$ Alexander A. W. Peters ${ }^{1} \cdot$ Frank W. Jansen ${ }^{1} \cdot$ Cornelis D. de Kroon $^{1}$. \\ Carina G. J. M. Hilders ${ }^{2}$
}

Received: 7 March 2018 / Accepted: 22 August 2018 / Published online: 14 September 2018

(c) The Author(s) 2018

\begin{abstract}
Purpose To evaluate the effectiveness of ovarian transposition (OT) prior to radiation therapy (RT) and to evaluate the effect of age on ovarian survival (OS) after OT.

Methods We performed a retrospective control study, with women (aged $<45$ years) who underwent OT prior to pelvic radiation, versus women diagnosed with cervical cancer and treated with hysterectomy/trachelectomy and radiation therapy. All women were treated between 1989 and 2010. The 5 years OS rate was calculated, with a sub-analysis for age (25-30; 31-35 and 36-40 years). Ovarian failure was defined as climacteric complaints (with or without starting hormone replacement therapy) and/or laboratory measurements (FSH $>40 \mathrm{IU} / \mathrm{L}$ and/or estradiol $<100 \mathrm{pmol} / \mathrm{L}$ ), or bilateral salpingo oophorectomy. Women were censored at recurrence.

Results Twenty-seven women after OT and 29 controls were included. The radiation dose was $44.8 \mathrm{~Gy}(25.0-63.0 \mathrm{~Gy})$ and $46.3 \mathrm{~Gy}(45.0-50.0 \mathrm{~Gy})$, respectively. The 5-year ovarian survival rate was $60.3 \%$ versus controls $0 \%(p<0.00195 \%$ CI 3.48-11.50). Despite the decrease in ovarian survival after OT with increasing age, in all age groups (25-30, 30-35 and 35-40) ovarian survival after OT was significantly better compared to women without OT ( $p=0.001 ; p=0.004$ and $p=0.000$, respectively). Neither intra-vaginal radiation therapy of concomitant chemotherapy in addition to pelvic radiation significantly altered ovarian survival.

Conclusions Our data shows that ovarian transposition prior to pelvic radiation is effective in women until the age of 35 years and needs to be discussed in patients aged 36-40 years.
\end{abstract}

Keywords Ovarian preservation · Ovarian transposition · Radiation therapy

\section{Introduction}

Premature ovarian insufficiency (POI) after high gonadotoxic therapy is one of the side effects of cancer treatment with high impact on quality of life. Due to the continued refinements in treatment regimens, the rates of complete remission and cure have been improved. Thereby, quality of life after cancer is becoming a very important issue in a large number of women [1]. For example in the Netherlands, around 350 new cases of fertile patients are diagnosed with

Ellen J. Hoekman

e.j.hoekman@lumc.nl

1 Department of gynecology, Leiden University Medical Center, P/O Box 9600, 2300RC Leiden, The Netherlands

2 Department of Gynecology, Reinier de Graaf Gasthuis Delft, Delft, The Netherlands cervical cancer every year [2]. Furthermore besides cervical cancer, patients with Hodgkin's disease, rectal cancer and other malignancies who need pelvic irradiation are at high risk where a small dose of irradiation can already cause infertility and POI $[3,4]$.

Infertility, climacteric symptoms such as vasomotor hot flashes, urogenital and sexual dysfunction and emotional disturbances are the side effects of POI with high impact on quality of life [5, 6]. Especially, estrogen withdrawal in young women is associated with decreased bone mineral density and impaired lipid profile with increased risk of ischemic heart disease [7, 8]. Although hormone replacement therapy is highly effective in counteracting these risks, long-term studies equating endogenous and exogenous estrogens are lacking [9]. Moreover, hormone replacement therapy (HRT) in women with POI may raise certain serious adverse side effects [10]. Finally, the success of this 
therapy depends largely on the patient's compliance of the prescribed protocol [11]. As it has been shown, a small number of women follow their HRT regularly [12].

In an attempt to protect the ovaries from pelvic irradiation and prevent POI, ovaries can be transposed outside the radiation field, also known as ovarian transposition (OT). This surgical technique has been performed since 1952, with varying rates of success [13]. The last 30 years, POI after transposition ranges from 33 to $100 \%$, using various techniques and modes of follow-up [14-28]. One reason of ovarian failure after OT may be the influence of scattered radiation therapy [29]. Moreover, it has been postulated that decreased blood flow to the ovaries, due to bending of the vessels as a result of the transposition, damage during surgery or as a side effect of the radiation therapy, increases the risk of POI. With regard to side effects of OT, the incidence of symptomatic ovarian cysts after OT is reported to range between 0 and 34\% [15, 19, 22, 27]. In some of the reported cases, removal of the transposed ovary was necessary. More disturbingly, several studies have reported ovarian metastases in transposed ovaries [30,31]. Thus, whether transposing the ovaries prior to radiation is a safe and effective procedure remains an ongoing issue.

We conducted a retrospective case-control study to evaluate the effectiveness and complications of ovarian transpositions prior to radiation therapy. Moreover, we evaluated the effect of age on ovarian survival after ovarian transposition.

\section{Materials and methods}

From hospital databases of the Leiden University Medical Center, all patients were selected in whom ovarian transposition was performed between 1988 and 2010. From the same databases in which data were entered prospectively, women eligible for the control group were selected: pre-menopausal cervical cancer patients in whom the ovaries were not transposed prior to pelvic radiation.

\section{Data collection}

Medical records of both cases and controls were reviewed for cancer treatment, survival, relapse and adjuvant cancer treatment. Since our study is retrospective by design, there was no obligation to have the protocol approved by our institutional review board.

With regard to ovarian survival, climacteric symptoms (irregular or no bleeding pattern, hot flushes), the use of hormonal replacement therapy and laboratory values (FSH and estradiol) were evaluated whenever available. Finally, complications of OT were assessed. Ovarian survival was defined as the absence of ovarian failure. Ovarian insufficiency was defined as follows: whenever at least two of the following criteria were reported, women were considered to suffer from ovarian failure: (1) climacteric symptoms, (2) use of hormonal substitution, (3) menopausal laboratory value (i.c. FSH $>40 \mathrm{IU} / \mathrm{mL}$ and/or estradiol $<100 \mathrm{pmol} / \mathrm{L}$ ). Patients with normal ovarian function were censored at the date of the last follow-up with explicit data on the abovementioned criteria for ovarian survival or at the date of systemic high gonadotoxic treatment for recurrence or metastatic disease. Follow-up of the ovarian function was continued until the first date of POI or in case of ovarian survival until a maximum of 5 years (when possible). Follow-up was reviewed until January 2018.

\section{Statistical analysis}

The collected data was analyzed using SPPS $20^{\circledR}$ (SPSS Inc, Chicago, IL, USA). Patient characteristics were compared with an independent $T$ test. Overall ovarian survival was estimated by the Kaplan-Meier method, and the log-rank test was used to compare ovarian survival between cases and controls, the influence of age, additional chemotherapy and/or intra-vaginal radiation therapy. The Mann-Whitney $U$ was performed to detect the difference of time until POI between OT and controls. With regard to age, the effect of RT after OT on ovarian survival was estimated for three different groups of age: 25-30 years, 31-35 years and 36-40 years. Figures were generated by Prism GraphPad 5 (GraphPad Software Inc., La Jolla, CA, USA).

\section{Results}

The follow-up of 27 patients who underwent OT prior to pelvic radiation were reviewed (OT group). The majority of patients suffered from cervical squamous cell carcinoma. Other patients suffered from rectal adenocarcinoma $(n=3$, stage 2, 3 and 3 ) or schwannoma ( $n=1$, grade II). Twentynine patients who suffered from cervical carcinoma and treated with pelvic radiation therapy ( 7 patients with adenocarcinoma, 22 patients with squamous cell carcinoma) in whom OT was not performed were included in the control group. In all women except one case (concomitant chemotherapy and pelvic radiation), surgery was the primary therapy. Characteristics, including details on adjuvant radiation therapy and concomitant chemotherapy of both cases and controls, are shown in Table 1.

In most patients, standard follow-up treatment was performed according to the Dutch national guidelines for cancer treatment. The follow-up, for example of cervical cancer patients consists of a 3 -month interval in the first 2 years, 6-month interval at year 3 and 4 and a 1 -year interval at year 5. Patients were seen earlier on request or when there were complaints. 
Table 1 Patient characteristics

\begin{tabular}{|c|c|c|c|}
\hline & Transposition & Control & $p$ value \\
\hline Mean age (years) & 33.4 & 36.67 & 0.02 \\
\hline $25-30$ & 9 & 4 & \\
\hline $31-35$ & 7 & 10 & \\
\hline $36-40$ & 8 & 8 & \\
\hline $40-44$ & 3 & 7 & \\
\hline \multicolumn{4}{|l|}{ Primary cancer } \\
\hline Cervical cancer & 23 & 29 & \\
\hline Rectal cancer & 3 & 0 & \\
\hline Schwannoma & 1 & 0 & \\
\hline \multicolumn{4}{|l|}{ FIGO } \\
\hline $1 b$ & 15 & 25 & \\
\hline $2 \mathrm{a}$ & 6 & 3 & \\
\hline $2 b$ & 2 & 1 & \\
\hline Other & 4 & 0 & \\
\hline Lymph node status & & & NS \\
\hline $\mathrm{LN}+$ & 8 & 12 & \\
\hline LN- & 19 & 17 & \\
\hline \multicolumn{4}{|l|}{ Post-operative therapy } \\
\hline $\begin{array}{l}\text { Mean external radiation dose } \\
\text { (Gy) }\end{array}$ & 44.88 & 46.32 & NS \\
\hline Mean internal radiation dose (Gy) & 16.93 & 13.89 & 0.021 \\
\hline Chemoradiation treatment & 9 & 0 & \\
\hline Adnex in situ & & & NS \\
\hline 1 adnexa & 5 & 3 & \\
\hline 2 adnexa & 22 & 26 & \\
\hline Disease recurrence & 5 & 5 & \\
\hline Diseased & 3 & 5 & \\
\hline
\end{tabular}

$L N$ lymph node, $N S$ non-significant

\section{Surgical procedure of OT}

Ovarian transposition was performed during laparotomy $(n=18,66.7 \%)$ for primary cancer treatment or by laparoscopy scheduled especially for OT $(n=9,33.3 \%)$. In all surgical reports, the normal appearance of the ovaries was mentioned. The proprial ligament and fallopian tube were ligated and the ovarian vascularity in the infundibulopelvic ligament was dissected upward to allow transposition of the ovary to the upper abdomen above the level of the umbilicus. The ovary, along with the attached fallopian tube, was attached to the fascia of the anterior abdominal wall with a non-absorbable suture. No additional measurements were performed to reduce the risk of ovarian torsion. In 21 patients, the right ovary was transposed, while the left ovary was transposed in four patients and in two other patients both ovaries were transposed. In one patient, data about the transposition site were not known. The decision of transposing one ovary instead of two was due to uncertainty of additional risks such as the formation of cysts and torsion at the time.

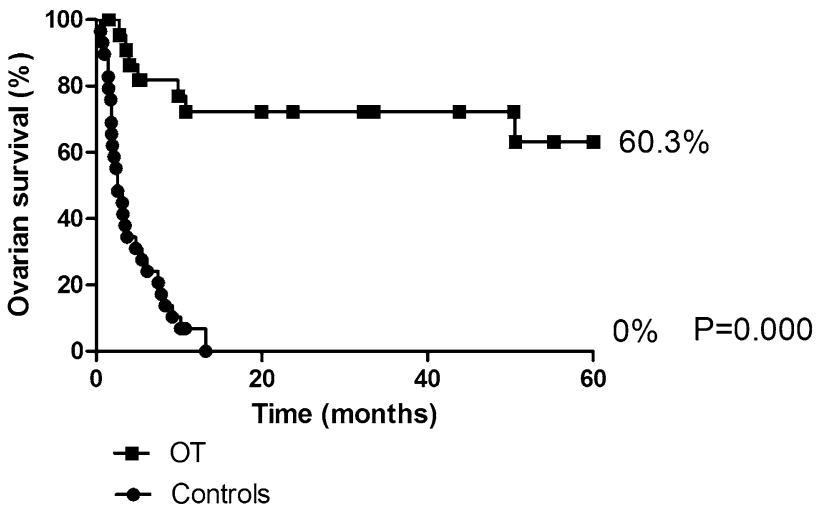

Fig. 15 years ovarian survival, case and controls

\section{Additional cancer treatment}

All patients received external pelvic radiation therapy with a mean dose of 44.8 Gy (range 25-63) in the OT group and 46.3 Gy (range 45-50) in the control group (NS). Ten patients of the OT group and 17 patients of the control group received additional brachytherapy (16.93 versus $13.89 \mathrm{~Gy}$, $p<0.05$ ) (Table 1). Nine patients received additional chemotherapy, mono or combined therapy of cisplatin, taxol and/ or topotecan, respectively.

\section{Ovarian survival}

The mean follow-up of OT patients, from RT until the latest date of normal ovarian function, was 34.5 months (min. 1.5-96.0).

In three cases, no details on ovarian function could be obtained for the medical records. These women were not included in the calculation of ovarian survival. One patient with cervical cancer did not experience menopausal complaints, despite elevated serum FSH (62 and $75 \mathrm{IU} / \mathrm{mL})$ at two different measurements: although she strictly did not fulfill the criteria for ovarian failure, she was considered to be suffering from ovarian insufficiency from the date of the first elevated serum FSH.

Eight $(29.6 \%)$ women in the OT group suffered from ovarian failure 5 years after pelvic radiation. Ovarian survival at 5 years was $60.3 \%$ (OT group) versus $0.0 \%$ (controls) $(p<0.001,(p<0.00095 \%$ CI 3.48-11.50) (Fig. 1). Median time to ovarian failure after radiation therapy was significantly shorter in the control group, 2.6 months, versus cases, 7.49 months $(p=0.009)$. Four out of the total of eight patients in whom OT failed suffered from ovarian failure within 6 months after radiation therapy. In two patients, the transposed ovaries (both ovarian survival at the time of surgery) were removed after, respectively, 2 (due to pseudomyxoma peritonei) and 45 months (preventive surgery 


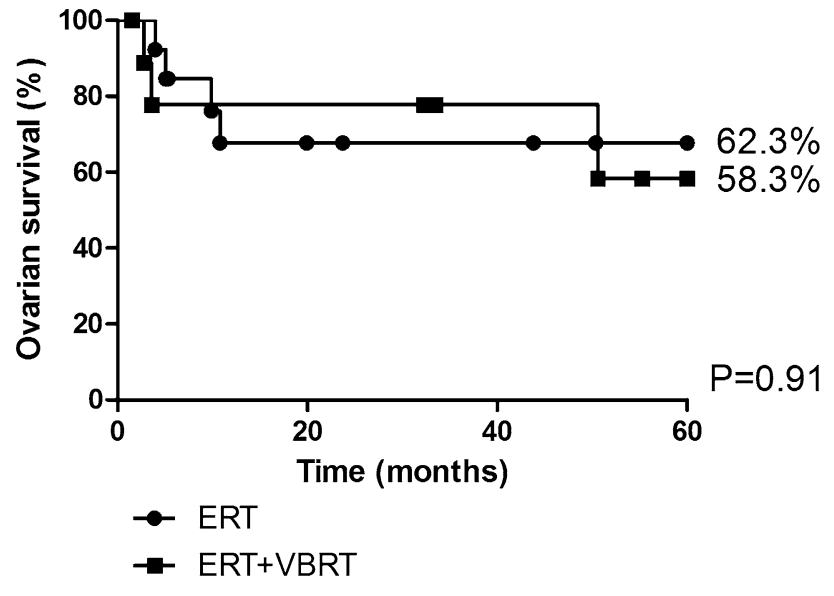

Fig. 2 Ovarian survival after OT with or without intra-vaginal radiation therapy

because of MSH-6 mutation). These cases were censored for ovarian survival analyses at the date of surgery.

Of the 24 patients in the OT group included in the ovarian survival analyses, 10 patients received external and intravaginal radiation therapy. Additional vaginal brachytherapy showed no significant difference in ovarian survival versus patients who received external radiation therapy alone: 5 years ovarian survival 58.3 versus $62.3 \%(p=0.91,95 \% \mathrm{CI}$ 33.15-52.94, Fig. 2). Within the control group, the addition of vaginal brachytherapy did not show a significant difference in ovarian survival after 5 years either: the median time until ovarian failure after OT was 2.4 years without brachytherapy (95\% CI $0.197-4.54$ ) versus 2.6 years after the addition of brachytherapy (95\% CI 1.24-3.96) ( $p=0.501)$.

Nine patients received chemotherapy, of which two patients received chemotherapy after ovarian failure, one patient received $80 \mathrm{mg} / \mathrm{kg}$ cisplatin and was lost to followup, and another patient received taxol, ifosfamide and cisplatin and was censored at the start of the chemotherapy. The other five patients were included in the ovarian survival analyses and received concomitant pelvic radiation and chemotherapy (four patients received five or six doses of cisplatin $40 \mathrm{mg} / \mathrm{m}^{2}$ weekly schedule, and one patient received cisplatin $40 \mathrm{mg} / \mathrm{m}^{2}$ and topotecan weekly schedule). There was no significant difference in the ovarian survival of transposed ovaries receiving chemoradiation or radiation therapy alone, although the number of patients was low in this analysis $(p=0.41)$.

There was a non-significant decrease in 5 years ovarian survival after OT with increasing age ( $p=0.16$, Fig. 3 ). Despite the decrease in ovarian survival after OT with increasing age, in all age groups (25-30, 30-35 and 35-40) ovarian survival after OT was significantly better compared to women without OT ( $p=0.001 ; p=0.004$ and $p=0.000$, respectively (Fig. 3a-c). Although ovarian survival was
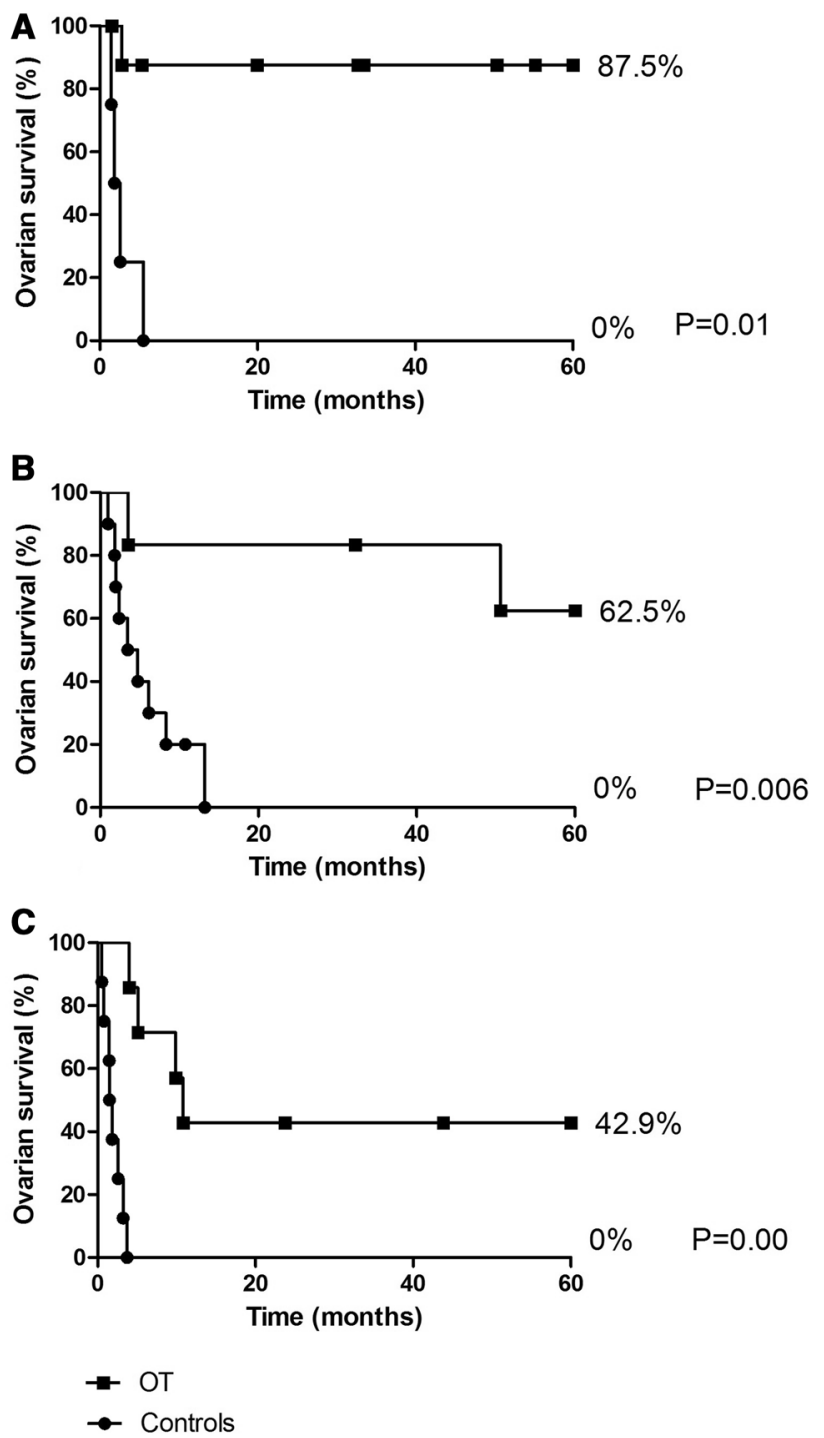

A. Ovarian survival age 25-30

B. Ovarian survival age $31-35$

C. Ovarian survival age $36-40$

Fig. 3 5-years ovarian survival after OT for different age groups

significantly longer after OT in all the groups, when considering OT in women aged 35-40 years, we have to consider the small proportion of women aged 35-40 years who benefited from OT (two out of eight patients, Table 2).

In three patients, OT was performed at the age of 42 years: two of these patients were among the three women who were lost to follow-up and consequently not included in the survival analysis; the other 42-year-old woman had normal ovarian function 5 years after OT.

None of the patients had become pregnant during followup, and high technological surrogacy was not performed. 
Table 2 Patient characteristics of patients after ovarian transposition and aged 35-40 years

\begin{tabular}{llllll}
\hline Nr. & Age at RT & $\begin{array}{l}\text { Ovarian } \\
\text { survival }\end{array}$ & Time (months) & Treatment & Notes \\
\hline 1 & 35.7 & Yes & 74.7 & ERT & None \\
2 & 37.6 & Yes & 23.7 & ERT & None \\
3 & 37.1 & Yes & 43.8 & ERT & BSO \\
4 & 35.7 & No & 5.1 & ERT & 1 ovary in situ \\
5 & 35.8 & No & 10.8 & ERT & Transposi- \\
& & & & & tion of two \\
& & & 18.7 & ERT & BSO \\
6 & 38.1 & No & 3.9 & ERT & None \\
7 & 38.6 & No & 9.9 & ERT and Cisplatin & None \\
8 & 35.7 & No & & &
\end{tabular}

\section{Complications}

All nine laparoscopic surgeries specially performed for ovarian transposition were uneventful. One ovarian torsion requiring oophorectomy of the transposed ovary (OT performed by laparotomy during primary surgery) was recorded. However, before oophorectomy, the patient was already diagnosed with POI despite OT. In none of the cases in this series, ovarian metastases were diagnosed during follow-up.

\section{Discussion}

Our data show that ovarian transposition (OT) prior to pelvic radiation results in a significant increase in ovarian survival after pelvic radiation: 5 years ovarian survival $60.3 \%$ after OT versus $0.0 \%$ without OT $(p<0.001)$ Published rates of POI and complications after OT prior to pelvic radiation differ greatly (ovarian survival between $33 \%$ and 100\%), which results in an ongoing debate about the safety and effectiveness of the procedure [14-28, 32]. However, most of the published data suffer from methodological flaws, since most studies are case series and non-comparative. Therefore, the results of effectiveness or disadvantages are not conclusive. The ovarian survival rates reported in this comparative study strongly support the advocates of ovarian transposition given its significant increase in ovarian survival after pelvic radiation and low complication rate.

Our results show that additional intra-vaginal radiation therapy and/or low dose chemotherapy (as part of chemoradiation therapy) has no significant impact on ovarian survival. Hence, these women should not be excluded from the option of OT. Unfortunately, due to small numbers it was not possible to compare patients after OT, receiving chemotherapy, EBRT and VBRT $(n=4)$, versus patients receiving EBRT and VBRT (without chemotherapy, $n=4$ ). The PORTEC-2 trial showed the efficacy and reduced side effects of VBRT compared with external beam pelvic radiotherapy (EBRT) for patients with high-intermediate risk of endometrial cancer. Additionally, patients receiving VBRT only reported less bowel symptoms, which provided a better quality of life than after EBRT, contributing to the theory that VBRT delivers less scatter radiation that results in less impact on the surrounding tissue [33]. In contrast to our findings, Morice et all reported ovarian survival of $100 \%$ after OT, $90 \%$ after OT and brachytherapy and $60 \%$ after OT, brachytherapy and external radiation therapy [19]. Furthermore, ovarian survival after OT, external radiation therapy and brachytherapy has been described, respectively, in 33\%; 80\% and 100\% [34-36]. Yamamoto reported a 5 year ovarian survival rate of $38.5 \%$ after OTs and radiation therapy [16]. Furthermore, Beukers reported ovarian survival in $41 \%$ of patients after OT and radiation therapy, with a mean follow-up of 43 months [26]. Hence there is no evidence to supports not transposing ovaries in case of pelvic chemoradiation in combination with intra-vaginal brachytherapy.

In the literature, the age above 40 years has often been used as a cutoff point. In our cohort three women older than 40 years underwent OT, of whom two were lost to followup. Hence, it is uncertain whether OT of women above the age of 40 years is worthwhile. Additionally, the ovarian survival was statistically significant in all age groups (Fig. 3), although on having a close look into the age group of 35-40 years only two out of eight patients (25\%) experienced ovarian survival until 74 months (Table 2).

Previous studies have shown a correlation between age and the dose of pelvic radiation and ovarian failure. Hamish et al. described ovarian failure at the age of 10 years after exposure to $18.4 \mathrm{~Gy}$, but at the age of 30 years ovarian failure occurred after exposure to $14.3 \mathrm{~Gy}$ [4]. The effect of OT in women above 40 years of age is limited, because of reduced fertilization and a higher risk of premature ovarian failure despite OT [19]. In addition, progressively smaller doses of chemotherapy are required to produce ovarian failure with increasing age [37-39]. In our opinion, to prevent POI, ovarian transposition should be 
discussed with all patients below the age of 35 years who need external radiation to the pelvis.

Despite OT, some women still suffer from ovarian failure after pelvic radiation. It has been shown that, due to scattered radiation, a substantial loss of ovarian function might still occur despite OT [21, 24, 40]. Furthermore, POI after OT and radiation therapy can be caused by migration of the ovaries back into the small pelvis after OT. Williams et al. reported ovarian failure which accounted in most patients of ovaries migrating back to the radiation area, as observed at repeat surgery [40]. An option for detecting the position of the ovaries is to attach a radio-opaque marker to the ovaries during transposition. The ovaries can be identified by imaging done for radiation planning and allows the radiotherapist to adjust the radiation field when necessary. Within our series, not all transposed ovaries were clipped by radio-opaque markers. Thus, in some cases it is unclear whether the ovary was still positioned outside the radiation field or had migrated back to the pelvis. In this context, we advise using radioopaque markers to visualize the ovaries prior to and during radiation therapy. Finally, even in women after OT without radiation therapy, POI has been reported in up to $7 \%$ of women $[15,17,18,26]$. Thus apart from damage due to radiation, multiple factors may contribute to failure of OT prior to radiation therapy.

Advocates of OT are supported by the data that POI has a high impact on the quality of life and may even cause more stress to the particular woman than suffering from cancer $[25,26]$. Therefore, it is worthwhile to prevent POI and preserve ovarian function and/or fertility. However, complications due to OT should be weighted. The major adverse events related to OT are, however, rare: the occurrence of ovarian cysts, ovarian torsion and ovarian metastases on the transposed ovary [19, 20, 23]. In the presented series, adverse events were rare: an ovarian torsion was reported in the transposition group and was surgically removed. No ovarian metastases were reported in this study and surgical procedures were uneventful. In our opinion, OT is a safe procedure and should be offered to women below 40 years old whenever pelvic radiation is indicated.

In conclusion, our data show that ovarian transposition prior to pelvic radiation is effective, since both ovarian survival $(p<0.001)$ and time to POI $(p<0.01)$ are significantly better after OT prior to radiation therapy. Despite the low complication risk of ovarian transposition, we advise only ovarian transposition in women until the age of 35 years prior to pelvic radiation to prevent POI with its disadvantageous impact on the quality of life. In women aged $35-40$ years, OT can be discussed when the procedure is executed during primary cancer surgery; however, the chances of long-term ovarian survival are limited.
Acknowledgements The authors thank A Sister's Hope and DSW Health Insurance, The Netherlands, for financial support.

Author contributions EJH, AAWP, CGJMH and CDdK conceived the study design. All authors, including EJH, CDdK, DK, AAWP, FWJ and CGJMH, collected data. EJH and DK performed data analysis and interpretation, generated figures and searched literature. All authors, including EJH, CDdK, DK, AAWP, FWJ and CGJMH, were involved in writing the paper and gave final approval of the submitted and published versions.

\section{Compliance with ethical standards}

Conflict of interest statement The authors declare no competing interests; all have read and approved the manuscript and agreed to its submission. The manuscript is an original work and not under consideration for publication elsewhere. The guidelines of the STROBE statement have been followed in the preparation of the manuscript.

Ethical approval We used data from official medical records and all participants were anonymous.

Open Access This article is distributed under the terms of the Creative Commons Attribution 4.0 International License (http://creativeco mmons.org/licenses/by/4.0/), which permits unrestricted use, distribution, and reproduction in any medium, provided you give appropriate credit to the original author(s) and the source, provide a link to the Creative Commons license, and indicate if changes were made.

\section{References}

1. Howard-Anderson J, Ganz PA, Bower JE, Stanton AL (2012) Quality of life, fertility concerns, and behavioral health outcomes in younger breast cancer survivors: a systematic review. J Natl Cancer Inst 104:386-405

2. Dutch Cancer Centers (2010) http://www.iknl.nl. Accessed 31 Dec 2010

3. Wallace WH, Thomson AB, Kelsey TW (2003) The radiosensitivity of the human oocyte. Hum Reprod 18:117-121

4. Wallace WH, Thomson AB, Saran F, Kelsey TW (2005) Predicting age of ovarian failure after radiation to a field that includes the ovaries. Int J Radiat Oncol Biol Phys 62:738-744

5. Absolom K, Eiser C, Turner L et al (2008) Ovarian failure following cancer treatment: current management and quality of life. Hum Reprod 23:2506-2512

6. Canada AL, Schover LR (2012) The psychosocial impact of interrupted childbearing in long-term female cancer survivors. Psychooncology 21:134-143

7. Davies MC, Hall ML, Jacobs HS (1990) Bone mineral loss in young women with amenorrhoea. BMJ 301:790-793

8. Lubiszewska B, Kruk M, Broda G et al (2012) The impact of early menopause on risk of coronary artery disease (PREmature Coronary Artery Disease In Women-PRECADIW case-control study). Eur J Prev Cardiol 19:95-101

9. McKinney KA, Thompson W (1998) A practical guide to prescribing hormone replacement therapy. Drugs 56:49-57

10. Shumaker SA, Legault C, Rapp SR et al (2003) Estrogen plus progestin and the incidence of dementia and mild cognitive impairment in postmenopausal women: the Women's Health Initiative Memory Study: a randomized controlled trial. JAMA 289:2651-2662 
11. Buist DS, Newton KM, Miglioretti DL et al (2004) Hormone therapy prescribing patterns in the United States. Obstet Gynecol 104:1042-1050

12. Cano A (1994) Compliance to hormone replacement therapy in menopausal women controlled in a third level academic centre. Maturitas 20:91-99

13. Batten R, Brown DE (1956) Protection of ovaries from radiation. Lancet 270:939-940

14. Al-Badawi IA, Al-Aker M, AlSubhi J et al (2010) Laparoscopic ovarian transposition before pelvic irradiation: a Saudi tertiary center experience. Int J Gynecol Cancer 20:1082-1086

15. Pahisa J, Martinez-Roman S, Martinez-Zamora MA et al (2008) Laparoscopic ovarian transposition in patients with early cervical cancer. Int J Gynecol Cancer 18:584-589

16. Yamamoto R, Okamoto K, Yukiharu T et al (2001) A study of risk factors for ovarian metastases in stage Ib-IIIb cervical carcinoma and analysis of ovarian function after a transposition. Gynecol Oncol 82:312-316

17. Van Eijkeren MA, Van DWI, El Sharouni SY, Heintz AP (1999) Benefits and side effects of lateral ovarian transposition (LOT) performed during radical hysterectomy and pelvic lymphadenectomy for early stage cervical cancer. Int J Gynecol Cancer 9:396-400

18. Thomas PR, Winstanly D, Peckham MJ, Austin DE, Murray MA, Jacobs HS (1976) Reproductive and endocrine function in patients with Hodgkin's disease: effects of oophoropexy and irradiation. Br J Cancer 33:226-231

19. Morice P, Juncker L, Rey A, El-Hassan J, Haie-Meder C, Castaigne D (2000) Ovarian transposition for patients with cervical carcinoma treated by radiosurgical combination. Fertil Steril 74:743-748

20. Morice P, Castaigne D, Haie-Meder C et al (1998) Laparoscopic ovarian transposition for pelvic malignancies: indications and functional outcomes. Fertil Steril 70:956-960

21. Feeney DD, Moore DH, Look KY, Stehman FB, Sutton GP (1995) The fate of the ovaries after radical hysterectomy and ovarian transposition. Gynecol Oncol 56:3-7

22. Husseinzadeh N, van Aken ML, Aron B (1994) Ovarian transposition in young patients with invasive cervical cancer receiving radiation therapy. Int J Gynecol Cancer 4:61-65

23. Anderson B, LaPolla J, Turner D, Chapman G, Buller R (1993) Ovarian transposition in cervical cancer. Gynecol Oncol 49:206-214

24. Owens S, Roberts WS, Fiorica JV, Hoffman MS, LaPolla JP, Cavanagh D (1989) Ovarian management at the time of radical hysterectomy for cancer of the cervix. Gynecol Oncol 35:349-351

25. Bilek K, Leitsmann H (1984) Transposition of the ovaries for function preservation in radical surgery and post-irradiation of cervical carcinoma. Zentralbl Gynakol 106:152-159

26. Buekers TE, Anderson B, Sorosky JI, Buller RE (2001) Ovarian function after surgical treatment for cervical cancer. Gynecol Oncol 80:85-88
27. Chambers SK, Chambers JT, Kier R, Peschel RE (1991) Sequelae of lateral ovarian transposition in irradiated cervical cancer patients. Int J Radiat Oncol Biol Phys 20:1305-1308

28. Hodel K, Rich WM, Austin P, DiSaia PJ (1982) The role of ovarian transposition in conservation of ovarian function in radical hysterectomy followed by pelvic radiation. Gynecol Oncol 13:195-202

29. van Beurden M, Schuster-Uitterhoeve AL, Lammes FB (1990) Feasibility of transposition of the ovaries in the surgical and radiotherapeutical treatment of cervical cancer. Eur J Surg Oncol $16: 141-146$

30. Morice P, Haie-Meder C, Pautier P, Lhomme C, Castaigne D (2001) Ovarian metastasis on transposed ovary in patients treated for squamous cell carcinoma of the uterine cervix: report of two cases and surgical implications. Gynecol Oncol 83:605-607

31. Sanjuan A, Roman SM, Martinez-Zamora MA, Pahisa J (2007) Bilateral ovarian metastasis on transposed ovaries from cervical carcinoma. Int J Gynecol Obstet 99:64-65

32. Gareer W, Gad Z, Gareer H (2011) Needle oophoropexy: a new simple technique for ovarian transposition prior to pelvic irradiation. Surg Endosc 7:2241-2246

33. Nout RA, Putter H, Jurgenliemk-Schulz IM et al (2012) Five-year quality of life of endometrial cancer patients treated in the randomised Post Operative Radiation Therapy in Endometrial Cancer (PORTEC-2) trial and comparison with norm data. Eur J Cancer 48:1638-1648

34. Stockle E, Verdier G, Thomas L et al (1996) Functional outcome of laparoscopically transposed ovaries in the multidisciplinary treatment of cervical cancers. Analysis of risk factors. J Gynecol Obstet Biol Reprod (Paris) 25:244-252

35. Bidzinski M, Lemieszczuk B, Zielinski J (1993) Evaluation of the hormonal function and features of the ultrasound picture of transposed ovary in cervical cancer patients after surgery and pelvic irradiation. Eur J Gynaecol Oncol 14(Suppl):77-80

36. Ploch E, Kietlinska Z, Kawczynska M (1988) Evaluation of the hormonal function of transposed ovaries in cervical cancer patients after surgery and pelvic irradiation. Preliminary report. Eur J Gynaecol Oncol 9:479-484

37. Goodwin PJ, Ennis M, Pritchard KI, Trudeau M, Hood N (1999) Risk of menopause during the first year after breast cancer diagnosis. J Clin Oncol 17:2365-2370

38. Whitehead E, Shalet SM, Blackledge G, Todd I, Crowther D, Beardwell CG (1983) The effect of combination chemotherapy on ovarian function in women treated for Hodgkin's disease. Cancer 52:988-993

39. Warne GL, Fairley KF, Hobbs JB, Martin FI (1973) Cyclophosphamide-induced ovarian failure. N Engl J Med 289:1159-1162

40. Williams RS, Littell RD, Mendenhall NP (1999) Laparoscopic oophoropexy and ovarian function in the treatment of Hodgkin disease. Cancer 86:2138-2142 